\title{
Necesidades de los docentes de Cartagena para introducir el patrimonio en el aula
}

\author{
Álvaro CHAPARRO SAINZ \\ Juan PARRA MARTÍNEZ
}

\section{Datos de contacto: \\ Álvaro Chaparro Sainz Universidad de Almería alvarocs@ual.es \\ Juan Parra Martínez Universidad de Murcia j.parramartinez@um.es}

Recibido: 24/09/2021

Aceptado: 01/12/2021

\begin{abstract}
RESUMEN
Recientemente numerosos estudios han confirmado la excelente valoración que el futuro profesorado posee acerca del uso de elementos patrimoniales en los contextos educativos. En este marco, se considera preciso descubrir cuáles son las necesidades que presenta el profesorado en activo acerca de este recurso para su introducción en el aula. Así, el objetivo principal de la presente investigación reside en conocer las demandas del profesorado en ejercicio, en concreto del entorno del Campo de Cartagena, para introducir el patrimonio en sus clases. Para llevar a cabo la investigación se ha utilizado un cuestionario, implementado anteriormente en estudios similares (Castro \& López-Facal, 2019), que ha sido respondido por docentes de etapas educativas preuniversitarias $(n=44)$. Para ello, se ha utilizado un diseño cuantitativo no experimental, con recopilación de información a través de un cuestionario con una escala Likert (1-5), cuyos datos se han analizado con el programa estadístico SPSS v.25. Entre los resultados más destacados, señalamos la existencia de divergencias significativas en las respuestas de unas etapas educativas respecto de otras, si bien el conjunto del profesorado reclama la necesidad de contar con ayuda de los organismos a través de la facilitación de materiales.
\end{abstract}

PALABRAS CLAVE: Educación patrimonial; Educación formal; Profesorado; Educación Infantil; Educación Primaria; Educación Secundaria. 


\title{
Needs of Cartagena teachers to introduce heritage in the classroom
}

\begin{abstract}
Recently, numerous studies have confirmed the excellent assessment that future teachers have about the use of heritage elements in educational contexts. In this term, it is considered necessary to find out what the needs of teachers in active teaching about this resource are for their introduction in the classroom. Therefore, the main objective of this research is to know the demands of teachers in practice, specifically the environment of the Campo de Cartagena, to introduce heritage in their classes. The aim is to answer the following question: what are the needs of teachers in terms of heritage education? To carry out the research, a questionnaire has been used, previously implemented in similar studies (Castro \& López-Facal, 2019), which has been answered by teachers from all the pre-university educational stages $(n=44)$. For this purpose, a nonexperimental quantitative design has been used, with information collected through a questionnaire with a Likert scale (1-5), whose data have been analyzed with the SPSS v.25 statistical program. Among the most noteworthy results, there are differences in the responses of some groups with respect to others. In this sense, while primary education teachers place emphasis on specific training, the creation of materials, the holding of theoretical and practical days and the implementation of a heritage education program; The teachers of Early Childhood Education and Secondary Education take a less favorable position.
\end{abstract}

KEYWORDS: Heritage education; Formal education; Teachers; Early Childhood Education; Primary Education; Secondary Education

\section{Introducción}

Las investigaciones realizadas en relación con las percepciones del profesorado en formación sobre la utilización de elementos patrimoniales en los procesos de enseñanza-aprendizaje de contenidos de índole histórica han confirmado la valoración positiva de los docentes sobre su incorporación en las aulas (Castro et al., 2020; Chaparro \& Felices, 2019; Felices et al., 2020; Gómez et al., 2020; Miralles et al., 2017; Trabajo \& Cuenca, 2020). La razón que explica la positiva evaluación del patrimonio como recurso docente reside en su capacidad para cambiar los procesos de enseñanza y de renovar el escenario metodológico y epistemológico del aprendizaje de las Ciencias Sociales (Calaf, 2010; Cuenca et al., 2018; Domínguez \& López-Facal, 2014; Estepa, 2013; Fontal, 2003; Fontal \& Gómez-Redondo, 2016; Martín \& Cuenca, 2011; Pinto, 2013; Soininen, 2017; Vicent et al., 2015).

Con todo, pese a las positivas valoraciones, los futuros docentes aseguran tener carencias a la hora de incorporar los recursos patrimoniales en los procesos educativos, mostrando a su parecer limitaciones metodológicas respecto a cómo se pueden utilizar (Chaparro \& Felices, 2019; Felices et al., 2020; Molina \& Muñoz, 2016; 
Moreno-Vera y Ponsoda-López de Atalaya, 2018). Un enunciado que concuerda con las conclusiones de algunos autores que muestran la escasa presencia del patrimonio en la formación inicial de los futuros docentes, así como la necesidad de formar al alumnado de los grados universitarios en educación patrimonial (Domínguez \& López; 2017; Fontal et al., 2017).

Este diagnóstico, circunscrito a los docentes en formación, invita a los autores a un cuestionamiento que supere estas etapas formativas y que se haga extensible al profesorado en activo. La posibilidad de investigar sobre el profesorado en ejercicio, al que se le atribuyen concepciones tradicionales del patrimonio (Estepa et al., 2008; Pinto, 2016), permite detectar in situ las necesidades que les demandan los procesos de enseñanza-aprendizaje. Ante esta situación, creemos que la investigación sobre las necesidades de estos maestros y maestras resulta fundamental. No en vano, sólo a través de este conocimiento basado en experiencias reales y en situaciones concretas podemos tener elementos de juicio que repercutan en un cambio metodológico y epistemológico (Gómez et al., 2020; Pinto \& Zarbato, 2017; Sánchez-Macías et al., 2019).

En el seno de una sociedad democrática se entiende primordial un adecuado conocimiento, uso y aprovechamiento del patrimonio en los contextos formativos. Una utilización del recurso construida y fundamentada sobre la base de una formación adecuada del profesorado. No en vano, los docentes son los encargados de implementar este recurso en los espacios educativos, así como de concienciar sobre la importancia que tiene el patrimonio dentro de nuestras sociedades. En este sentido, como señalan Trabajo y Cuenca (2017), la educación patrimonial en torno a la enseñanza de las Ciencias Sociales favorece la adquisición de capacidades y competencias que permiten al alumnado desarrollarse como ciudadanos responsables, con hábitos de vida favorables con su entorno y con una capacidad de acción crítica sobre el medio.

Además, un correcto uso del patrimonio posibilita la consolidación de un sentimiento de identidad, facilitando el apuntalamiento de los valores de una sociedad necesitada de sentimientos de solidaridad, igualdad, empatía y respeto tanto por lo propio, como por lo ajeno (Cuenca et al., 2020; Lucas \& Delgado-Algarra, 2021; Rico, 2004). Así, la educación patrimonial se convierte en un elemento clave para el desarrollo de un niño o de una niña en su formación integral dentro de la sociedad (Delgado-Algarra \& Cuenca, 2020; Munilla \& Marín-Cepeda, 2020; Pérez, 2013). De hecho, existe una mayor probabilidad de que una persona que haya recibido una educación patrimonial adecuada consiga desarrollarse de forma holística e integral que una persona cuyo conocimiento y valoración del patrimonio sea escaso (Cuenca, 2014).

En este contexto de necesidad, la creación y evaluación de planes formativos centrados en la enseñanza de las Ciencias Sociales a través del patrimonio se ha aseverado como una medida absolutamente necesaria (Fontal et al., 2019; Fontal \& Martínez-Rodríguez, 2017). En este sentido, desde un punto de vista formativo, se pretende, a través de este proceso, dar respuesta a las problemáticas identificadas en relación con la inserción de este recurso en el aula (Calaf et al., 2017; Castro \& LópezFacal, 2019; Estepa et al., 2007). Con todo, consideramos fundamental poner el prisma de la investigación en el profesorado en activo, en aquel que se encuentra, en estos 
momentos, actuando en el aula y, en consecuencia, haciendo frente a las dificultades propias de la enseñanza. Por ello, hemos optado por preguntar directamente a los agentes activos sobre sus particulares necesidades a la hora de actuar en el aula y en el momento de enfrentarse a las dinámicas inherentes a la práctica educativa.

En definitiva, la presente investigación parte de los planteamientos y preguntas planteadas por Castro y López-Facal (2019) en una investigación en la que se trataban de identificar las principales necesidades que los docentes de dos centros educativos de la provincia de A Coruña presentaban a la hora de introducir en su aula elementos de carácter patrimonial para la enseñanza de sus contenidos. Según los autores, los resultados más relevantes del estudio confirmaban la necesidad de formación específica en educación patrimonial, así como de contar con materiales específicos para trabajar sobre el patrimonio local.

En el presente artículo, partiendo por tanto de los enunciados del estudio mencionado, se replica dicha investigación en el entorno de Cartagena (Murcia, España), un espacio marcado por una contrastada presencia de elementos patrimoniales, sean materiales, inmateriales como naturales, lo que justifica este planteamiento científico. En este contexto, estos bienes, de valor incalculable para el conocimiento histórico no sólo de la comarca sino también del país deberían, a nuestro parecer, tener una presencia equitativa en los centros educativos (Chaparro et al., 2018; Chaparro et al., 2019; Morales et al., 2017). Por esta razón, recuperamos la pregunta que formulaban Castro y López-Facal (2019) en su investigación: ¿cuáles son las necesidades percibidas por el profesorado en cuanto a la educación patrimonial?

\section{Método}

\section{Objetivos}

En este artículo se desarrolla una investigación preliminar exploratoria con carácter de evaluación diagnóstica. Se trata de un estudio, de carácter cuantitativo, que posee, como objetivo general, conocer las necesidades del profesorado en activo del entorno del Campo de Cartagena para introducir el patrimonio en los contextos educativos formales. De la misma manera, se concretan dos objetivos específicos:

- OE1. Diagnosticar si alguna de las actuaciones e intervenciones demandadas debe ser desarrollada en las titulaciones universitarias de formación del profesorado.

- OE2. Recabar información sobre las acciones que deban ser desarrolladas en los procesos formativos de los futuros docentes de toda etapa educativa.

\section{Instrumento}

Se ha utilizado un diseño cuantitativo no experimental, con recopilación de información a través de un cuestionario con escala Likert (1-5). Se eligió este tipo de diseño porque es capaz de responder a los problemas tanto en términos descriptivos como en relación con las variables, lo que garantiza el rigor de los datos obtenidos (Hernández \& Maquilón, 2010). El instrumento para la recogida de información es un cuestionario - validado por expertos -, titulado "Cuestionario sobre Educación 
Patrimonial" (Castro \& López-Facal, 2019).

La escala está conformada por dos bloques claramente diferenciados: uno destinado a la identificación del participante y un segundo bloque que se encuentra, a su vez, dividido en cuatro dimensiones: Formación y renovaciones metodológicas (1), Papel que juega la familia y el entorno (2), Metodología y actividades (3) y, por último, Resultados esperados (4).

\section{Tabla 1}

Ítems del cuestionario utilizado

Ítem Enunciado del ítem

1.1. Contar con formación específica en educación patrimonial ayudaría a mi labor docente.

1.2. Unas jornadas teóricas y prácticas sobre educación patrimonial serían de gran ayuda para mi formación.

1.3. Me gustaría contar con materiales de trabajo específicos para la educación patrimonial.

1.4. Realizar un programa de educación patrimonial en el que participe todo el centro me parece una buena idea.

1.5. Integrar el estudio del patrimonio en el currículo ayudaría a organizar mis clases.

1.6. Resultaría muy positiva la colaboración entre materias para el trabajo con el patrimonio.

2.1. Que los ayuntamientos, museos y diversos organismos ofreciesen al centro materiales con los que poder trabajar el patrimonio local sería muy positivo.

2.2. Sería muy positivo que las familias se implicasen en el trabajo del centro sobre patrimonio (búsqueda de información, entrevistas, coloquios, etc.)

2.3. Sería muy positivo que los vecinos y vecinas colaborasen con el trabajo del centro sobre patrimonio (entrevistas, historias de vida, relatos, etc.)

3.1. Me parecería buena idea que diferentes organizaciones y personalidades se acercasen al centro para realizar actividades de educación patrimonial.

3.2. Me parecería buena idea hacer salidas con el alumnado a diversas organizaciones e instituciones para trabajar con el patrimonio.

3.3. Resultaría muy efectivo realizar talleres, charlas, etc. sobre educación patrimonial con personas externas al centro.

3.4. Resultaría muy efectivo que el alumnado hiciese trabajos de investigación sobre patrimonio.

3.5. Considero que las salidas del centro para realizar trabajos de campo sobre el patrimonio local serían muy efectivas.

3.6 Realizar trabajos de creación de materiales sobre el patrimonio con el alumnado sería muy positivo.

4.1. Trabajando el patrimonio local se contribuye a que el alumnado conozca más lo propio.

4.2. Trabajando el patrimonio local se conseguiría una difusión de la cultura de la localidad.

4.3. Trabajando el patrimonio local se conseguiría que el alumnado valorase su entorno.

4.4. Trabajando con el patrimonio local el alumnado se identificaría con su cultura y se sensibilizaría con ella. 
Para estimar la fiabilidad del bloque de preguntas analizado, empleamos el método de consistencia interna basado en el Alfa de Cronbach que permite estimar la fiabilidad de un instrumento de medida compuesto por un conjunto de ítems de tipo escala Likert (1-5). De esta manera, los ítems son sumables en una puntuación única que mide un rasgo que es importante en la construcción teórica del instrumento. La fiabilidad de la escala debe obtenerse siempre con los datos de cada muestra para garantizar la medida fiable del constructo en la muestra concreta de la investigación. Así, el cuestionario utilizado obtiene un índice de Alfa de Cronbach de, 750, lo que se considera aceptable ( $\alpha>$.7) (Frías-Navarro, 2020).

\section{Participantes}

En relación con la muestra de la investigación $(n=44)$ cabe indicar que es de carácter no probabilístico por conveniencia, ya que ha sido seleccionada en atención al criterio de la accesibilidad a los sujetos y de la adecuación a los objetivos de la investigación (McMillan \& Schumacher, 2005). La muestra con la que se ha realizado el estudio recoge las opiniones de profesorado en activo de diferentes etapas educativas: Educación Infantil (7), Educación Primaria (25) y Educación Secundaria (12), que imparten docencia en centros educativos del Campo de Cartagena.

\section{Procedimiento y análisis de datos}

Para proceder con la recogida de información, se envió un email a docentes de diferentes centros educativos del entorno del Campo de Cartagena. El correo electrónico contenía una carta, en la que se explicaban los objetivos del proyecto, al tiempo que se les facilitaba un enlace web a través del cual poder participar. Las fechas entre las que se produjo la recogida de opiniones fue entre el 1 de octubre y el 31 de diciembre del 2020.

El análisis cuantitativo de los datos se realizó con el programa estadístico SPSS, en su versión v25.0, lo que posibilitó realizar un análisis completo de la información recogida. En este sentido, el análisis de los datos se ha realizado en tres fases diferentes: a) un análisis de los estadísticos descriptivos de los ítems, b) un estudio de las frecuencias en las respuestas al cuestionario $\mathrm{y}$, por último, c) un análisis de los resultados en relación con la variable "etapa educativa".

\section{Resultados}

Atendiendo a los resultados de los estadísticos descriptivos de los ítems, observamos (Tabla 2) cómo el ítem (2.1) que presenta una valoración de su media más elevada $(4,70)$ y que, al tiempo, posee la desviación típica más baja $(0,509)$ del conjunto es el ítem que indica: Que los ayuntamientos, museos y diversos organismos ofreciesen al centro materiales con los que poder trabajar el patrimonio local sería muy positivo. Es decir, el profesorado consultado nos muestra su convencimiento unánime acerca de la 
necesidad de contar con más recursos y materiales que les permita aprovechar convenientemente el patrimonio local.

En segundo lugar, con una valoración en ambos casos de 4,68 identificamos dos ítems que se cuestionan en torno a los logros que se obtendrían en caso de trabajar el patrimonio en el aula. De este modo, en el ítem 4.3 se señala cómo Trabajando el patrimonio local se conseguiría que el alumnado valorase su entorno, mientras que el ítem 4.4 subraya que Trabajando con el patrimonio local el alumnado se identificaría con su cultura y se sensibilizaría con ella. De este modo, inferimos cómo el profesorado vincula la enseñanza del patrimonio con el entorno del alumnado y con su identificación en él; es decir, los docentes consultados vinculan el uso del patrimonio local con el mejor conocimiento del espacio en el que vive y se desarrolla el alumnado.

Por último, en tercer lugar, encontramos el ítem 3.2, titulado: Me parecería buena idea hacer salidas con el alumnado a diversas organizaciones e instituciones para trabajar con el patrimonio, que recibe una valoración de 4,64, misma valoración que recibe el ítem 4.2 relativo a Trabajando el patrimonio local se conseguiría una difusión de la cultura de la localidad. Estas opiniones ratifican el uso de espacios exteriores para conocer el patrimonio, un binomio (exterior y patrimonio) que generalmente va de la mano, si bien se reclama, nuevamente, la colaboración de instituciones públicas para un conocimiento del entorno más próximo al alumnado.

\section{Tabla 2}

Descriptivos, media de los ítems y desviación típica

\begin{tabular}{|c|c|c|c|}
\hline Dimensión & Ítem & Media & Desviación típica \\
\hline \multirow{6}{*}{$\begin{array}{l}\text { Formación y } \\
\text { renovaciones } \\
\text { metodológicas }\end{array}$} & 1.1 & 4,07 & 0,759 \\
\hline & 1.2 & 4,25 & 0,719 \\
\hline & 1.3 & 4,16 & 0,861 \\
\hline & 1.4 & 4,45 & 0,791 \\
\hline & 1.5 & 3,95 & 1,056 \\
\hline & 1.6 & 4,23 & 0,743 \\
\hline \multirow{3}{*}{$\begin{array}{lll}\text { Familia } & \text { y } & \text { el } \\
\text { entorno } & & \end{array}$} & 2.1 & 4,70 & 0,509 \\
\hline & 2.2 & 4,34 & 0,776 \\
\hline & 2.3 & 4,25 & 0,719 \\
\hline \multirow{6}{*}{$\begin{array}{l}\text { Metodología y } \\
\text { actividades }\end{array}$} & 3.1 & 4,52 & 0,590 \\
\hline & 3.2 & 4,64 & 0,574 \\
\hline & 3.3 & 4,52 & 0,590 \\
\hline & 3.4 & 4,25 & 0,943 \\
\hline & 3.5 & 4,43 & 0,846 \\
\hline & 3.6 & 4,41 & 0,757 \\
\hline \multirow{4}{*}{$\begin{array}{l}\text { Resultados } \\
\text { esperados }\end{array}$} & 4.1 & 4,61 & 0,579 \\
\hline & 4.2 & 4,64 & 0,574 \\
\hline & 4.3 & 4,68 & 0,518 \\
\hline & 4.4 & 4,68 & 0,518 \\
\hline
\end{tabular}


En el lado opuesto, el ítem 1.5 donde se menciona que Integrar el estudio del patrimonio en el currículo ayudaría a organizar mis clases, ha recibido la media de valoración más baja en el conjunto de ítems $(3,95)$. Este dato nos muestra cómo, en esta ocasión, el problema de la integración del patrimonio en el proceso de enseñanzaaprendizaje no reside, por tanto, en las limitaciones del texto normativo como quizás en otras circunstancias anteriormente señaladas.

En esta línea, cabe destacar cómo el ítem 1.1 donde se valora, por parte del profesorado, la necesidad de Contar con formación específica en educación patrimonial ayudaría a mi labor docente, ha recibido una consideración de 4,07 sobre cinco, una de las más bajas del conjunto. Ante este resultado, cabe la posibilidad de realizarse algún cuestionamiento, como por ejemplo: ¿hasta qué punto una propuesta formativa paliaría las necesidades del profesorado? o ¿es necesario concebir una formación para ayudar a los docentes?

\section{Figura 1}

Descriptivos de los ítems analizados

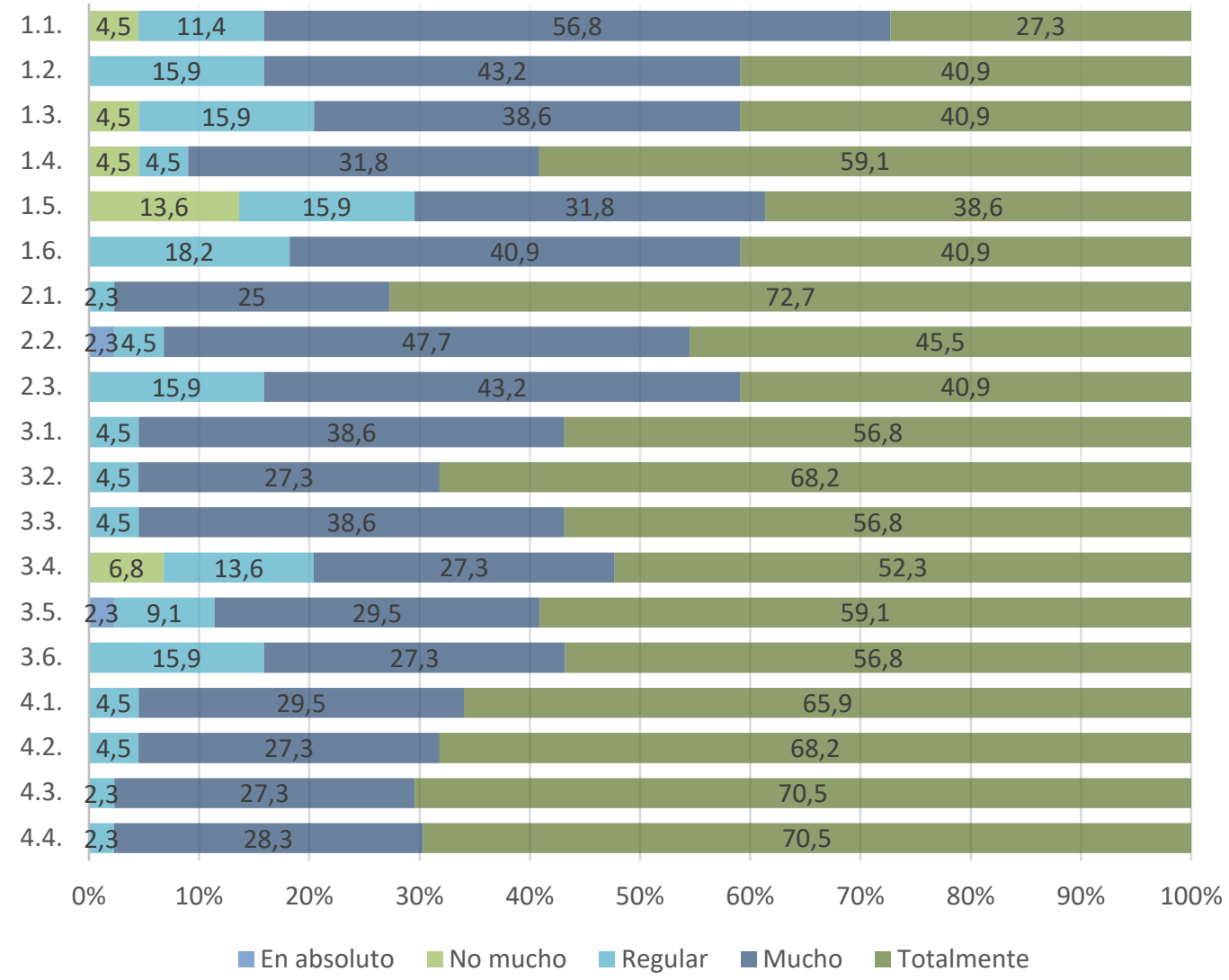


En una segunda fase, profundizando en la investigación, se desarrolló un análisis de frecuencias. Así, como se observa en la figura 1, tres ítems poseyeron mayor unanimidad en sus respuestas, dado que más del setenta por ciento de los participantes en la investigación respondieron "totalmente" a los enunciados planteados en las encuestas. De este modo, el ítem que mostró mayor unanimidad por parte de los participantes en la encuesta fue el relativo a: Que los ayuntamientos, museos y diversos organismos ofreciesen al centro materiales con los que poder trabajar el patrimonio local sería muy positivo (ítem 2.1), donde el 72,7\% (n=32) de las personas encuestadas opinó estar completamente de acuerdo con el enunciado. En esta línea, se mostraron las respuestas a los ítems 4.3 y 4.4. donde el 70,5\% $(n=31)$ de los participantes valoró como "totalmente" sus enunciados.

En el lado opuesto, el ítem que peor puntuación recibió fue el ítem 1.5 donde se mencionaba que Integrar el estudio del patrimonio en el currículo ayudaría a organizar mis clases dado que el 13,6\% $(n=6)$ de los participantes no se mostró muy de acuerdo con este enunciado, mientras que un 15,9\% $(\mathrm{n}=7)$ lo valoró como "regular". Al mismo tiempo, un 6,8\% (n=3) calificó el ítem 3.4 relativo a Resultaría muy efectivo que el alumnado hiciese trabajos de investigación sobre patrimonio como "no mucho", lo que da muestra de las dudas que generó entre los participantes en la encuesta. De hecho, sobre este mismo ítem, un 13,6\% (n=6) valoró como "regular" esta consideración.

Por último, en una tercera fase del estudio se realizó un análisis de los resultados descriptivos obtenidos en función de la variable dependiente "etapa educativa", que señalaba la etapa en la que impartían docencia los participantes en la investigación. Así, 7 miembros de la muestra, es decir, un 15,9\% impartían magisterio en Educación Infantil; mientras que 25 participantes de la muestra, es decir, un 56,8\% eran docentes de Educación Primaria; y que, finalmente, 12 intervinientes en la investigación eran profesores de Educación Secundaria, es decir, un 27,3\% de la muestra.

Atendiendo a las cuatro dimensiones del instrumento de investigación utilizado: Formación y renovaciones metodológicas (1), Papel que juega la familia y el entorno (2), Metodología y actividades (3) y Resultados esperados (4), se ha procedido a la realización de un análisis atendiendo a dicha variable dependiente. En este sentido, como veremos, las medias de los participantes que imparten docencia en Educación Infantil realizan generalmente valoraciones más bajas a los enunciados respecto al resto de docentes. De hecho, de los 19 ítems analizados, las respuestas de los docentes de Educación Infantil han mostrados índices medios inferiores al resto en 17 ocasiones.

En cuanto a la dimensión 1 (Tabla 3), observamos cómo existe una diversidad de opiniones destacada en torno al ítem 1.5, Integrar el estudio del patrimonio en el currículo ayudaría a organizar mis clases, ya que se produce, incluso, una diferencia de casi un punto entre la opinión de los docentes de Educación Primaria y los de Educación Secundaria. Mientras que los maestros y maestras de Educación Primaria valoran con un 4,32 la introducción del patrimonio en el currículo, los docentes de Educación Secundaria, que valoran el ítem con un 3,33, no consideran esta acción relevante para la organización de sus clases en relación con el patrimonio. 


\section{Tabla 3}

Descriptivos, media de los ítems y desviación típica de la dimensión 1

Dimensión 1: Formación y renovaciones metodológicas

\begin{tabular}{|c|c|c|c|c|c|}
\hline Ítem & Factor & $\mathbf{N}$ & Media & L. inferior & L. superior \\
\hline \multirow{4}{*}{1.1} & E. Infantil & 7 & 3,57 & 2,84 & 4,30 \\
\hline & E. Primaria & 25 & 4,28 & 4,00 & 4,56 \\
\hline & E. Secundaria & 12 & 3,92 & 3,41 & 4,42 \\
\hline & Total & 44 & 4,07 & 3,84 & 4,30 \\
\hline \multirow{4}{*}{1.2} & E. Infantil & 7 & 3,71 & 3,26 & 4,17 \\
\hline & E. Primaria & 25 & 4,48 & 4,21 & 4,75 \\
\hline & E. Secundaria & 12 & 4,08 & 3,58 & 4,59 \\
\hline & Total & 44 & 4,25 & 4,03 & 4,47 \\
\hline \multirow{4}{*}{1.3} & E. Infantil & 7 & 3,71 & 3,26 & 4,17 \\
\hline & E. Primaria & 25 & 4,36 & 4,05 & 4,67 \\
\hline & E. Secundaria & 12 & 4,00 & 3,28 & 4,72 \\
\hline & Total & 44 & 4,16 & 3,90 & 4,42 \\
\hline \multirow{4}{*}{1.4} & E. Infantil & 7 & 4,14 & 3,50 & 4,78 \\
\hline & E. Primaria & 25 & 4,64 & 4,41 & 4,87 \\
\hline & E. Secundaria & 12 & 4,25 & 3,53 & 4,97 \\
\hline & Total & 44 & 4,45 & 4,21 & 4,70 \\
\hline \multirow{4}{*}{1.5} & E. Infantil & 7 & 3,71 & 3,26 & 4,17 \\
\hline & E. Primaria & 25 & 4,32 & 3,93 & 4,71 \\
\hline & E. Secundaria & 12 & 3,33 & 2,55 & 4,12 \\
\hline & Total & 44 & 3,95 & 3,63 & 4,28 \\
\hline \multirow{4}{*}{1.6} & E. Infantil & 7 & 3,86 & 3,51 & 4,21 \\
\hline & E. Primaria & 25 & 4,24 & 3,90 & 4,58 \\
\hline & E. Secundaria & 12 & 4,42 & 3,99 & 4,84 \\
\hline & Total & 44 & 4,23 & 4,00 & 4,45 \\
\hline
\end{tabular}

En cambio, el ítem que más homogeneidad muestra en sus respuestas es el 1.4, Realizar un programa de educación patrimonial en el que participe todo el centro me parece una buena idea, puesto que todas las respuestas se ubican en un valor igual o inferior a 0,5. De esta manera, el conjunto de la muestra revela un considerable interés por vincular al centro educativo a la hora de realizar un programa de educación patrimonial; si bien es el profesorado de Educación Primaria el que se muestra más proclive a dicha acción educativa $(4,64)$.

En relación con la dimensión 2 (Tabla 4), los datos nos muestran cómo el profesorado en activo de Educación Primaria $(4,84)$ y Educación Secundaria $(4,75)$ valora mejor que el profesorado en Educación Infantil $(4,14)$ el ítem 2.1., titulado Que los ayuntamientos, museos y diversos organismos ofreciesen al centro materiales con los que poder trabajar el patrimonio local sería muy positivo. Una diferencia en la valoración que puede estar relacionada, entre otros motivos, con las características 
formativas del profesorado, la disposición de tiempo o el encorsetamiento curricular.

\section{Tabla 4}

Descriptivos, media de los ítems y desviación típica de la dimensión 2

\begin{tabular}{|c|c|c|c|c|c|}
\hline \multicolumn{6}{|c|}{ Dimensión 2: Papel que juega la familia y el entorno } \\
\hline Ítem & Factor & $\mathbf{N}$ & Media & L. inferior & L. superior \\
\hline \multirow{4}{*}{2.1} & E. Infantil & 7 & 4,14 & 3,50 & 4,78 \\
\hline & E. Primaria & 25 & 4,84 & 4,69 & 4,99 \\
\hline & E. Secundaria & 12 & 4,75 & 4,46 & 5,04 \\
\hline & Total & 44 & 4,70 & 4,55 & 4,86 \\
\hline \multirow{4}{*}{2.2} & E. Infantil & 7 & 4,29 & 3,59 & 4,98 \\
\hline & E. Primaria & 25 & 4,48 & 4,27 & 4,69 \\
\hline & E. Secundaria & 12 & 4,08 & 3,34 & 4,82 \\
\hline & Total & 44 & 4,34 & 4,11 & 4,58 \\
\hline \multirow{4}{*}{2.3} & E. Infantil & 7 & 4,14 & 3,50 & 4,78 \\
\hline & E. Primaria & 25 & 4,20 & 3,88 & 4,52 \\
\hline & E. Secundaria & 12 & 4,42 & 3,99 & 4,84 \\
\hline & Total & 44 & 4,25 & 4,03 & 4,47 \\
\hline
\end{tabular}

En el lado opuesto, los docentes de las tres etapas educativas muestran una opinión similar en el ítem 2.3, en el que se enuncia que Sería muy positivo que los vecinos y vecinas colaborasen con el trabajo del centro sobre patrimonio (entrevistas, historias de vida, relatos, etc.). Una opinión favorable hacia la intervención en los procesos educativos de agentes externos al aula que se confirma si atendemos a los datos recogidos del ítem 2.2., titulado Sería muy positivo que las familias se implicasen en el trabajo del centro sobre patrimonio (búsqueda de información, entrevistas, coloquios, etc.). Sin embargo, sí se observa que en este último ítem la predisposición de los docentes de Educación Secundaria hacia la participación de parientes del alumnado en clase es más baja que en el resto de las etapas educativas.

Por lo que respecta a la dimensión 3 (Tabla 5), el análisis revela la existencia de una remarcable homogeneidad en la respuesta al ítem 3.1, titulado Me parecería buena idea que diferentes organizaciones y personalidades se acercasen al centro para realizar actividades de educación patrimonial. En este sentido, nuevamente, el profesorado reclama la colaboración de agentes externos al aula para la introducción de los elementos patrimoniales en sus dinámicas docentes. Igualmente, se ha observado una valoración positiva, en los tres casos, con respecto al ítem 3.5., titulado Considero que las salidas del centro para realizar trabajos de campo sobre el patrimonio local serían muy efectivas. Un enunciado que pone en relación una práctica habitual en los contextos educativos formales, como es el aprovechamiento directo del patrimonio en el espacio en el que se ubica, con la colaboración con profesionales ajenos al ejercicio docente, como se mencionaba en el anterior ítem. 


\section{Tabla 5}

Descriptivos, media de los ítems y desviación típica de la dimensión 3

\begin{tabular}{|c|c|c|c|c|c|}
\hline \multicolumn{6}{|c|}{ Dimensión 3: Metodologías y actividades } \\
\hline Ítem & Factor & $\mathbf{N}$ & Media & L. inferior & L. superior \\
\hline \multirow{4}{*}{3.1} & E. Infantil & 7 & 4,43 & 3,70 & 5,16 \\
\hline & E. Primaria & 25 & 4,52 & 4,31 & 4,73 \\
\hline & E. Secundaria & 12 & 4,58 & 4,16 & 5,01 \\
\hline & Total & 44 & 4,52 & 4,34 & 4,70 \\
\hline \multirow{4}{*}{3.2} & E. Infantil & 7 & 4,43 & 3,70 & 5,16 \\
\hline & E. Primaria & 25 & 4,72 & 4,53 & 4,91 \\
\hline & E. Secundaria & 12 & 4,58 & 4,16 & 5,01 \\
\hline & Total & 44 & 4,64 & 4,46 & 4,81 \\
\hline \multirow{4}{*}{3.3} & E. Infantil & 7 & 4,14 & 3,50 & 4,78 \\
\hline & E. Primaria & 25 & 4,64 & 4,44 & 4,84 \\
\hline & E. Secundaria & 12 & 4,50 & 4,07 & 4,93 \\
\hline & Total & 44 & 4,52 & 4,34 & 4,70 \\
\hline \multirow{4}{*}{3.4} & E. Infantil & 7 & 4,14 & 3,15 & 5,13 \\
\hline & E. Primaria & 25 & 4,16 & 3,74 & 4,58 \\
\hline & E. Secundaria & 12 & 4,50 & 4,07 & 4,93 \\
\hline & Total & 44 & 4,25 & 3,96 & 4,54 \\
\hline \multirow{4}{*}{3.5} & E. Infantil & 7 & 4,29 & 3,59 & 4,98 \\
\hline & E. Primaria & 25 & 4,44 & 4,04 & 4,84 \\
\hline & E. Secundaria & 12 & 4,50 & 4,07 & 4,93 \\
\hline & Total & 44 & 4,43 & 4,17 & 4,69 \\
\hline \multirow{4}{*}{3.6} & E. Infantil & 7 & 4,00 & 3,08 & 4,92 \\
\hline & E. Primaria & 25 & 4,52 & 4,25 & 4,79 \\
\hline & E. Secundaria & 12 & 4,42 & 3,91 & 4,92 \\
\hline & Total & 44 & 4,41 & 4,18 & 4,64 \\
\hline
\end{tabular}

Finalmente, en relación con la dimensión 4 (Tabla 6), resulta interesante destacar, particularmente, la homogeneidad en las opiniones del profesorado consultado acerca de los beneficios pedagógicos que se lograrían en el aula en caso de trabajar el patrimonio local, especialmente respecto a la mejora de la valoración de "lo propio" por parte del alumnado. En este sentido, el reconocimiento de elementos personales favorece la comprensión y la identificación de contextos pretéritos permitiendo de este modo un mejor aprendizaje de contenidos inicialmente abstractos para el alumnado.

Igualmente, en esta dimensión, se considera relevante remarcar la excelente opinión expresada por los docentes de Educación Primaria y Educación Secundaria respecto al ítem 4.4., titulado Trabajando con el patrimonio local el alumnado se identificaría con su cultura y se sensibilizaría con ella. De este juicio se valora, por tanto, el posicionamiento de los docentes ante los procesos de aculturación que se pueden producir en los contextos escolares. 


\section{Tabla 6}

Descriptivos, media de los ítems y desviación típica de la dimensión 4.

\begin{tabular}{llcccc}
\hline \multicolumn{5}{c}{ Dimensión 4: Resultados esperados } \\
\hline Ítem & \multicolumn{1}{c}{ Factor } & N & Media & L. inferior & L. superior \\
\hline \multirow{4}{*}{4.1} & E. Infantil & 7 & 4,43 & 3,70 & 5,16 \\
& E. Primaria & 25 & 4,68 & 4,48 & 4,88 \\
& E. Secundaria & 12 & 4,58 & 4,16 & 5,01 \\
& Total & 44 & 4,61 & 4,44 & 4,79 \\
\hline \multirow{4}{*}{4.2} & E. Infantil & 7 & 4,43 & 3,70 & 5,16 \\
& E. Primaria & 25 & 4,72 & 4,53 & 4,91 \\
& E. Secundaria & 12 & 4,58 & 4,16 & 5,01 \\
& Total & 44 & 4,64 & 4,46 & 4,81 \\
\hline \multirow{4}{*}{4.3} & E. Infantil & 7 & 4,29 & 3,59 & 4,98 \\
& E. Primaria & 25 & 4,80 & 4,63 & 4,97 \\
& E. Secundaria & 12 & 4,67 & 4,35 & 4,98 \\
& Total & 44 & 4,68 & 4,52 & 4,84 \\
\hline \multirow{4}{*}{4.4} & E. Infantil & 7 & 4,29 & 3,59 & 4,94 \\
& E. Primaria & 25 & 4,76 & 4,58 & 5,04 \\
& E. Secundaria & 12 & 4,75 & 4,46 & 4,84 \\
\hline
\end{tabular}

\section{Discusión}

Atendiendo a los resultados obtenidos se observa cómo el profesorado consultado valora positivamente la posibilidad de recibir ayuda para la integración del patrimonio en los procesos educativos, lo que concuerda con otros estudios (Castro \& López-Facal, 2019; Miralles et al., 2017). En esta misma línea, se observa cómo, de manera general, los encuestados valoran la intervención de las instituciones públicas en la creación de materiales; de hecho, los docentes en activo demandan que los organismos pongan a disposición de los centros educativos recursos, materiales y alternativas pedagógicas que puedan implementar en sus aulas, como se confirma en otros estudios (Castro \& López-Facal, 2019; Cambil \& Fernández, 2016). Esta situación nos lleva a nuevas preguntas: ¿están capacitados los docentes para la creación de sus propios materiales en materia patrimonial?, ¿la falta de tiempo que reclaman los docentes para la preparación de materiales puede explicar esta demanda?, ¿qué papel deben tener las instituciones públicas que gestionan elementos patrimoniales?, etc.

En cuanto al trabajo en el aula con el patrimonio local, las respuestas de los docentes muestran unos elevados índices de aceptación del uso de este recurso con el alumnado. Una valoración positiva que, nuevamente, coincide con los resultados obtenidos en otras investigaciones (Castro \& López-Facal, 2019; Molina \& Ortuño, 2017). En este sentido, se observa cómo el conjunto de docentes valora de un modo positivo que el alumnado tenga un conocimiento más profundo del entorno que le rodea, reclamando planes formativos que incidan en el trabajo del patrimonio local, especialmente en Educación Primaria. En esta misma etapa educativa se observa un 
menor interés por la realización de trabajos de investigación sobre el patrimonio por parte del alumnado, quizás debido al nivel cognitivo que se les presupone en torno a la cuestión y a la necesidad de mayores habilidades y conocimientos para desarrollar una investigación adecuada.

Sin embargo, en el lado opuesto, la necesidad menos valorada entre el profesorado consultado reside en la integración del estudio del patrimonio en el currículo. Este hecho puede tener su origen en el carácter disciplinar que se le otorga al patrimonio, especialmente vinculado con contenidos de ciencias sociales o ciencias naturales. Especialmente, si tenemos en cuenta que cada docente es el encargado de impartir su propia materia, lo que puede implicar que la inclusión del patrimonio en la ley no le afecte directamente en el momento de impartir su asignatura. Dicho esto, como es conocido, existen normativas educativas vigentes que recogen ampliamente una introducción de este recurso en las aulas de Educación Primaria (Fontal et al., 2017).

Una vez analizados y contrastados los resultados obtenidos consideramos relevante señalar una serie de posibles metas, planteadas a modo de discusión, que pudieran actuar en beneficio de la integración de los elementos patrimoniales en un aula de educación obligatoria: mejorar la relación existente entre los centros educativos y las diferentes instituciones que se encargan de la difusión del patrimonio, tales como museos y ayuntamientos; desarrollar la importancia de la educación patrimonial dentro de la formación docente; apoyar la sensibilización sobre el patrimonio local de los agentes de la comunidad educativa: alumnado, docentes y familias y; finalmente, dotar a los centros de materiales y orientaciones metodológicas que fomenten la difusión del patrimonio en las aulas.

\section{Conclusiones}

Los resultados obtenidos en la investigación muestran, a nuestro parecer, la relevancia de cuestionar al profesorado en activo sobre sus necesidades para incorporar elementos patrimoniales en el aula, en particular aquellos de índole local, considerados por los docentes consultados como trascendentes en los procesos de enseñanza-aprendizaje. Pese a esta concordancia, en términos generales, los docentes nos muestran divergencias en sus opiniones en función de la etapa educativa en la que ejercen. Habida cuenta, entre otras razones, de las diferencias estrategias metodológicas que se implementan en cada una de las tres mencionadas etapas: Educación Infantil, Educación Primaria y Educación Secundaria.

En términos generales, el profesorado en ejercicio reclama la participación de agentes externos a la acción docente del aula con el fin de enriquecerla y mejorarla. De hecho, de manera concisa, el profesorado reclama que los ayuntamientos, museos y diversos organismos ofrezcan al centro materiales con los que poder trabajar el patrimonio local, al tiempo que considera beneficioso hacer salidas con el alumnado a diversas organizaciones e instituciones para trabajar con el patrimonio. Es decir, reconocen la importancia de colaborar intensamente con agentes externos al centro educativo, especialmente si están vinculados con elementos relacionados con el aprovechamiento del patrimonio local.

De este modo, se considera que, más allá de establecer formaciones concretas a 
desarrollar en titulaciones universitarias (OE1) o procesos formativos de los futuros docentes (OE2), el profesorado en activo reclama acciones concretas (materiales, recursos, salidas, etc.) que establezcan sinergias entre los cuerpos educativos formales y las instituciones o agentes vinculados a la gestión, conservación y difusión del patrimonio. Con todo, esta situación nos invita a cuestionarnos acerca del porqué de estas respuestas, planteando como hipótesis, precisamente, la carencia de una formación específica en las etapas previas a la acción profesional.

A este respecto, si bien de manera general el profesorado acepta la activa participación de técnicos, especialistas, vecinos o familiares en sus contextos educativos; la percepción hacia cada uno de estos agentes externos varía en función del grupo de profesionales consultado. En este sentido, por ejemplo, la intervención familiar no recibe la misma valoración positiva por parte del profesorado de Educación Secundaria respecto de la opinión de otros cuerpos docentes. Igualmente, mientras que el profesorado de Educación Primaria pone en valor la formación específica, la creación de materiales, la celebración de jornadas teóricas y prácticas, así como la realización de un programa de educación patrimonial; las respuestas del profesorado de Educación Infantil y de Educación Secundaria nos muestran cómo sus percepciones están más alejadas siendo más negativas respecto de las emitidas por el profesorado de Educación Primaria.

Ante esta situación, consideramos que la formación de los docentes debe concebirse como un punto de reflexión básico y continuo en la progresiva mejora del sistema educativo. Así, de manera particular, esta reflexión debe permitirnos mejorar y acondicionar los mecanismos necesarios que posibiliten una correcta introducción de elementos patrimoniales en todos los contextos educativos formales. Sin embargo, las actuaciones venideras beneficiarían únicamente a quienes se formen en el futuro próximo en los espacios universitarios lo que, a su vez, plantea una duda con respecto al procedimiento a seguir con quienes en la actualidad se encuentran ejerciendo y no han recibido dicha formación.

En este marco deben ser destacadas las iniciativas formativas que están surgiendo para paliar todas las carencias detectadas en los contextos de aula preuniversitarios. Una de ellas, por ejemplo, es el programa formativo Patrimonializarte (Castro et al., 2021), el cual ha demostrado la pertinencia de este tipo de actuaciones ante los aprendizajes significativos del alumnado participante. Un proyecto, desarrollado en etapas educativas obligatorias, que se revela como una actuación necesaria dado que puede resolver un problema a docentes que no han recibido una formación completa en educación patrimonial. Al tiempo, este tipo de proyectos debe venir acompañado de otras soluciones, como la construcción de puentes entre todos los agentes que participan, de una u otra manera, en la administración, conservación y divulgación de los bienes patrimoniales. La búsqueda de sinergias que aglutinen a los diversos actores en un espacio, como el patrimonial, común a toda la sociedad se traduciría en mejoras en los procesos de enseñanza.

En definitiva, si bien esta investigación muestra limitaciones como consecuencia de su muestra $(n=44)$, razón por la que sus resultados no pueden generalizarse ni al conjunto de la Región de Murcia ni a otros espacios más amplios, sí consideramos que el profesorado consultado muestra una pauta sobre la que regresar en posteriores 
investigaciones. La detección de necesidades concretas permite el establecimiento de planes específicos que puedan solucionar problemas generales, puesto que la identificación de particularidades en espacios geográficos delimitados puede ser la vía para solucionar situaciones similares en otros contextos.

\section{Agradecimientos}

Este trabajo es resultado del proyecto de investigación "Conceptos metodológicos y métodos activos de aprendizaje para la mejora de las competencias docentes del profesorado" (PGC2018-094491-B-C33) (MCI/AEI/FEDER, UE), subvencionado por el Ministerio de Ciencia, Innovación y Universidades y cofinanciado con fondos FEDER de la UE; así como del proyecto de I+D+i "La enseñanza y el aprendizaje de competencias históricas en bachillerato: un reto para lograr una ciudadanía crítica y democrática" (PID2020-113453RB-I00), financiado por MCIN/AEI/10.13039/501100011033.

\section{Conflicto de intereses}

Los autores declaran no tener ningún conflicto de intereses. Los financiadores no tuvieron ningún papel en el diseño del estudio; en la recopilación, análisis o interpretación de datos; en la redacción del manuscrito, o en la decisión de publicar los resultados.

\section{Contribuciones de los autores}

Conceptualización, Á. C. S. y J. P. M.; metodología, Á. C. S.; análisis formal, Á. C. S.; investigación, J. P. M.; análisis de datos, Á. C. S.; redacción del borrador original, J. P. M.; redacción, revisión y edición, J. P. M.; supervisión, Á. C. S.

\section{Referencias}

Calaf, R. (2010). Un modelo de investigación didáctica del patrimonio. Enseñanza de las ciencias sociales: revista de investigación, 9, 17-28.

Calaf, R., San Fabián, J. L., y Gutiérrez, S. (2017). Evaluación de programas educativos en museos: Una nueva perspectiva. Bordón, 69(1), 45-65. http://dx.doi.org/10.13042/Bordon.2016.42686

Cambil, M. E., y Fernández, R. (2016). El concepto actual de Patrimonio Cultural y su valor educativo: fundamentación teórica y aplicación didáctica. En M. E. Cambil y A. Tudela (Eds.), Educación y patrimonio cultural: fundamentos, contextos y estrategias didácticas (pp. 27-46). Ediciones Pirámide.

Castro, L., y López-Facal, R. (2019). Educación patrimonial: necesidades sentidas por el profesorado de infantil, primaria y secundaria. Revista Interuniversitaria de Formación del Profesorado, 94(33.1), 97-114. https://doi.org/10.47553/rifop.v33i1.72020

Castro, L., Rodríguez-Medina, J., y López-Facal, R. (2021). Educación patrimonial para una ciudadanía participativa. Evaluación de resultados de aprendizaje del alumnado en el programa Patrimonializarte. Revista Electrónica Interuniversitaria de Formación del Profesorado, 92(1), 205-219. https://doi.org/10.6018/reifop.444881

Castro, B., Castro, L., Conde, J., y López-Facal, R. (2020). Concepciones del profesorado sobre el uso educativo del patrimonio. Revista Interuniversitaria de Formación 
del Profesorado, 34(3), 77-96. https://doi.org/10.47553/rifop.v34i3.81620

Chaparro, Á., y Felices, M. M. (2019). Percepciones del profesorado en formación inicial sobre el uso del patrimonio en contextos educativos. Revista Interuniversitaria de Formación del Profesorado, 33(3), 327-346. https://doi.org/10.9685/74264

Chaparro, Á., Felices, M. M., y Martínez, R. (2018). Aprender desde el pasado: usos del patrimonio histórico de Cartagena en Educación Secundaria y Bachillerato. En A. I. Ponce y J. Ortuño (Eds). Pensando el patrimonio: Usos y recursos en el ámbito educativo. Universidad de Murcia.

Chaparro, Á. Martínez, C., Moral, F. J., y Cespedosa, R. (2019). Desarrollar la conciencia histórica a través del patrimonio en el Grado en Educación Primaria. Revista Didáctica de las Ciencias Experimentales y Sociales, 36, 17-32.

Cuenca, J. M. (2014). El papel del patrimonio en los centros educativos: hacia la socialización patrimonial. Tejuelo, 19, 76-96.

Cuenca, J. M., Molina, S., y Martín, M. (2018). Identidad, ciudadanía y patrimonio. Análisis comparativo de su tratamiento didáctico en museos de Estados Unidos y España. Arbor. Revista de Ciencia, Pensamiento y Cultura, 194(788), 1-13. https://orcid.org/0000-0002-0190-5739

Cuenca, J. M., Martín, M., y Estepa, J. (2020). Buenas prácticas en educación patrimonial. Análisis de las conexiones entre emociones, territorio y ciudadanía. Aula Abierta, 49(1), 45-54. https://doi.org/10.17811/rifie.49.1.2020.45-54

Delgado-Algarra, E. J., y Cuenca, J. M. (2020). Challenges for the Construction of Identities With Historical Consciousness: Heritage Education and Citizenship Education. En E. J. Delgado-Algarra y J. M. Cuenca. (Eds.), Handbook of Research on Citizenship and Heritage Education (pp.1-15). IGI Global.

Domínguez, A., y López-Facal, R. (2017). Patrimonios en conflicto, competencias cívicas y formación profesional en educación primaria. Revista de Educación, 375, 86104.

Domínguez, A., y López-Facal, R. (2014). Patrimonio, paisaje y educación: Formación inicial del profesorado y educación cívica del alumnado de primaria. Clío: History and History Teaching, 40, 2-26.

Estepa, J. (2013). La educación patrimonial en la escuela y el museo: investigación y experiencias. Universidad de Huelva.

Estepa, J., Ávila, R., y Ferreras, M. (2008). Primary and secondary teachers' conceptions about heritage and heritage education: A comparative analysis. Teaching and Teacher Education, 24(8), 2095-2107. https://doi.org/10.1016/j.tate.2008.02.017

Estepa, J., Ávila, R., y Ruiz, R. (2007). Concepciones sobre la enseñanza y difusión del patrimonio en las instituciones educativas y los centros de interpretación: estudio descriptivo. Enseñanza de las ciencias sociales: revista de investigación, 6, 75-94.

Felices, M. M., Chaparro, Á., y Rodríguez, R. A. (2020). Perceptions on the use of heritage to teach history in Secondary Education teachers in training. Humanities and Social Sciences Communications, 7(123), 1-10.

Fontal, 0. (2003). La educación patrimonial. Teoría y práctica en el aula, el museo e Internet. Trea. 
Fontal, O., García, S., Arias, B., y Arias, V. (2019). Evaluación de la calidad de programas de educación patrimonial: construcción y calibración de la escala Q-Edutage. Revista de Psicodidáctica, 24(1), 31-38. https://doi.org/10.1016/j.psicod.2018.07.003

Fontal, 0., y Martínez-Rodríguez, M. (2017). Evaluación de programas educativos sobre Patrimonio Cultural Inmaterial. Estudios pedagógicos, 43(4), 69-89. http://dx.doi.org/10.4067/S0718-07052017000400004

Fontal, O., y Gómez-Redondo, C. (2016). Heritage Education and Heritagization Processes: SHEO Methodology for Educational Programs Evaluation. Interchange, 47(1), 65-90.

Fontal, O., Ibáñez, A., Martínez-Rodríguez, M., y Rivero, P. (2017). El patrimonio como contenido en la etapa de Primaria: del currículum a la formación de maestros. Revista Electrónica Interuniversitaria de Formación del Profesorado, 20(2), 79-95. https://doi.org/10.6018/reifop/20.2.286321

Frías-Navarro, D. (2020). Apuntes de consistencia interna de las puntuaciones de un instrumento de medida. Universidad de Valencia. Disponible en: https://www.uv.es/friasnav/AlfaCronbach.pdf

Gómez, C. J., Miralles, P., Fontal, O., y Ibáñez, A. (2020). Cultural Heritage and Methodological Approaches-An Analysis through Initial Training of History $\begin{array}{llll}\text { Teachers } \quad \text { (Spain-England). } & \text { Sustainability, }\end{array}$ https://doi.org/10.3390/su12030933

Hernández, F., y Maquilón, J. J. (2010). Introducción a los diseños de investigación educativa. En S. Nieto (Coord.). Principios, métodos y técnicas para la investigación educativa (pp. 110-126). Dyckinson.

Lucas, L., y Delgado-Algarra, E. J. (2021). Patrimonio y coeducación en el marco curricular de la educación primaria. Revista Interuniversitaria de Formación del Profesorado, 35(2), 65-82. https://doi.org/10.47553/rifop.v97i35.2.87585

Martín, M. J., y Cuenca, J. M. (2011). La enseñanza y el aprendizaje del patrimonio en los museos: la perspectiva de los gestores. Revista de Psicodidáctica, 16(1), 99-122.

McMillan, J. H., y Schumacher, S. (2005). Investigación educativa. (5aa ed). Pearson Educación.

Miralles, P., Gómez, C. J., y Rodríguez, R. A. (2017). Patrimonio, competencias históricas y metodologías activas de aprendizaje. Un análisis de las opiniones de los docentes en formación en España e Inglaterra. Estudios pedagógicos, 43(4), 161184. http://dx.doi.org/10.4067/S0718-07052017000400009.

Molina, S., y Muñoz, R. E. (2016). La opinión del profesorado de Educación Secundaria sobre el papel del patrimonio en la enseñanza formal de las ciencias sociales: un estudio de caso. Revista Complutense de Educación, 27(2), 863-880. https://doi.org/10.5209/rev RCED.2016.v27.n2.48411

Molina, S., y Ortuño, J. (2017). Concepciones del profesorado iberoamericano de Secundaria sobre la contribución del patrimonio local al desarrollo del pensamiento histórico. Estudios Pedagógicos, 43(4), 185-202. http://dx.doi.org/10.4067/S0718-07052017000400010

Morales, M. J., Arias, L. y Egea, A. (2017). El patrimonio y las fuentes materiales en la práctica docente de la Educación Primaria en la Región de Murcia. Hermus. 
Heritage \& Museography, 18, 102-115.

Moreno-Vera, J. R., y Ponsoda-López de Atalaya, S. (2018). La percepción del alumnado sobre la didáctica del patrimonio en la enseñanza de la Historia. En R. Roig-Vela, (Ed.). El compromiso académico y social a través de la investigación e innovación educativas en la Enseñanza Superior (pp. 317-325). Octaedro.

Munilla, C., y Marín-Cepeda, S. (2020). Patrimonio y emociones. Estado de la cuestión desde las perspectivas internacional y nacional. Revista Interuniversitaria de Formación del Profesorado, 34(1), 177-196. https://doi.org/10.47553/rifop.v34i1.74886

Pinto, H. (2013). Usos del patrimonio en la didáctica de la historia: perspectivas de alumnos y profesores portugueses relativas a identidad y conciencia histórica. Educatio Siglo XXI, 31(1), 61-88.

Pinto, H. (2016). Educação Histórica e patrimonial. En I. Barca y L. A. Marqués (Coords)., Educação Histórica: Perspetivas de Investigação Nacional $e$ Internacional (pp. 26-33). CITCEM.

Pinto, H., y Zarbato, J. (2017). Construyendo un aprendizaje significativo a través del patrimonio local: prácticas de Educación patrimonial en Portugal y Brasil. Estudios Pedagógicos, 43(4), 203-227. http://dx.doi.org/10.4067/S071807052017000400011

Rico, L. (2004). La difusión del patrimonio a través de las nuevas tecnologías: Nuevos entornos para la educación patrimonial histórico-artística. En M. I. Vera-Muñoz y D. Pérez (Coords.). Formación de la ciudadanía: las TICs y los nuevos problemas (pp. 1-13). Universidad de Alicante.

Sánchez-Macías, I., Fontal, O., y Rodríguez-Medina, J. (2019). La evaluación de Aprendizajes: una práctica pendiente en Educación Patrimonial. Revista Interuniversitaria de Formación del Profesorado, 33(2), 163-186. https://doi.org/10.47553/rifop.v33i2.73325

Soininen, T. (2017) Adopt-a-monument: preserving archaeological heritage for the people, with the people. Journal of Community Archaeology \& Heritage, 4(2), 131137.

Trabajo, M., y Cuenca, J. M. (2017). La educación patrimonial para la adquisición de competencias emocionales y territoriales del alumnado de enseñanza secundaria. Pulso: revista de educación, 40, 159-174.

Trabajo, M., y Cuenca, J. M. (2020). Student concepts after a didactic experiment in heritage education. Sustainability, 12(7), 3046. https://doi.org/10.3390/su12073046

Vicent, N., Ibáñez, A., y Asensio, M. (2015). Evaluación de programas de educación patrimonial de base tecnológica. Virtual Archaeology Review, 13(6), 18-25. 
